\title{
Author Correction: Postprandial glycaemic dips predict appetite and energy intake in healthy individuals
}

Patrick Wyatt (1), Sarah E. Berry, Graham Finlayson, Ruairi O'Driscoll (1D, George Hadjigeorgiou, David A. Drew, Haya Al Khatib, Long H. Nguyen (D), Inbar Linenberg, Andrew T. Chan, Tim D. Spector (1D, Paul W. Franks (1), Jonathan Wolf(D, John Blundell and Ana M. Valdes (iD

Correction to: Nature Metabolism https://doi.org/10.1038/s42255-021-00383-x, published online 12 April 2021.

In the version of this article initially published, in the labels at the bottom of Fig. 2d,e, 'mins' should have read 'kcal'. In Supplementary Tables 1 and 3, mean and SD values were incorrectly transposed. The errors have been corrected in the HTML and PDF versions of the article.

Published online: 13 July 2021

https://doi.org/10.1038/s42255-021-00436-1

(๑) The Author(s), under exclusive licence to Springer Nature Limited 2021 